\title{
A CONSISTENT EXTENSION OF CONDORCET'S ELECTION PRINCIPLE
}

\author{
H.P. Young* and A. Levenglick**
}

RR 77-15

July 1977

Presented at the ORSA/TIMS National Meeting at Miami, Florida, November 4, 1976. This work was supported in part by the National Science Foundation under Grant MCS 75-07414 A01 with the Graduate School of the City University of New York.

*Graduate School and University Center of the City University of New York, and IIASA.

**Graduate School and University Center of the City University of New York, and Equitable Life Assurance Society of America, New York.

Research Reports provide the formal record of research conducted by the International Institute for Applied Systems Analysis. They are carefully reviewed before publication and represent, in the Institute's best judgment, competent scientific work. Views or opinions expressed therein, however, do not necessarily reflect those of the National Member Organizations supporting the Institute or of the Institute itself.

International Institute for Applied Systems Analysis

A-2361 Laxenburg, Austria 



\section{PREFACE}

This paper is part of a larger task in Systems and Decision Sciences that is concerned with institutional structures and their role in shaping decisions. A particular problem in this field is to characterize the formal processes or rules by which groups of decision makers should choose among available alternatives. Typically, these rules are formulated as specific voting procedures. This article examines certain common-sense properties of voting rules and describes a particular rule that is shown to be the unique one satisfying these properties. The first half of the paper introduces the basic concepts and summarizes the results; the second half is devoted to a detailed proof, using convex analysis, of the principal theorem. 



\section{SUMMARY}

Voting rules on many alternatives may be broadly divided into two classes: those that use a scheme of "weighting" the alternatives to determine their overall order of desirability, and those that use binary comparison to ascertain whether there is an alternative (called a Condorcet alternative) that is able to defeat every other alternative by a simple majority. The first approach is identified with Borda, the second with Condorcet. In this paper it is shown that the basic desirable property of weighting systems namely "consistency" under aggregation - can be achieved without sacrificing the common-sense property of choosing a Condorcet alternative whenever one exists. In fact, these two properties, together with the requirement of "neutrality" on alternatives, essentially determine a unique rule known in the literature as Kemeny's rule. 



\section{A Consistent Extension of Condorcet's Election Principle}

\section{INTRODUCTION}

In France during the latter half of the eighteenth century, the problem of designing voting rules for an assembly began to be studied systematically for the first time. Two of the major contributors were Jean-Charles de Borda and Marie J.A.N. Caritat, Marquis de Condorcet, both members of l'Académie Royale des Sciences. From these two men sprang two streams of thought on the problem of collective decision making that persist to the present day. In 1770, Borda [4] read a paper before the Academy in which he proposed the following method, which he called "election by order of merit": for each voter's announced (linear) preference order on the alternatives, a score of 0 is assigned to the least preferred alternative, 1 to the next-to-least preferred, and so forth; then the total score of each alternative is computed and the one with the highest score is declared the winner. Condorcet [6] proposed instead that if there is some alternative (the condorcet alternative) that defeats every other in pairwise simple majority voting, then that alternative should be selected. A difficulty with Borda's rule is that it may not result in the choice of the condorcet alternative (as in Example 1 where $a_{1}$ is the Condorcet alternative and $a_{2}$, the Borda choice); but a difficulty with Condorcet's principle is that, if a Condorcet alternative does not exist, it is unclear which alternative should be chosen.

Example 1.

Number of voters

$\begin{array}{lll}a_{1} & a_{2} & a_{3} \\ a_{2} & a_{3} & a_{1} \\ a_{3} & a_{1} & a_{2}\end{array}$


The challenge of combining the regularity of Borda's approach with Condorcet's principle into a unified method is a long-standing problem in the theory of elections. Black [3] proposed that the condorcet alternative be chosen when one exists, and otherwise that the Borda method be reverted to. This somewhat ad hoc proposal avoids the fundamental issue of choosing properties that are natural in the context of election, and then asking what (if any) methods have these properties.

In this paper we shall identify certain basic properties suggested by the Borda and the condorcet approaches, respectively, and show that these properties uniquely determine a method first proposed by John Kemeny [10, 11].

Let $A=\left\{a_{1}, a_{2}, \ldots, a_{m}\right\}$ be a set of $m$ alternatives under consideration, $\mathbb{N}=\{0,1,2, \ldots\}$ the names of the possible voters. A preference order on A is simply a inear order. We will denote such an order by the sequence $\sigma=\left(a_{i_{1}}, a_{i_{2}}, \ldots, a_{i_{m}}\right)$ where $a_{i_{1}}$ is most preferred, and so forth. $L(A)$ will denote the set of all $\mathrm{m}$ ! linear orders on $\mathrm{A}$.

For any (finite) electorate $\underset{\sim}{\mathrm{M}} \underset{\sim}{\mathbb{N}} \underset{\sim}{\mathbb{N}}$ a profize on $\underset{\sim}{\mathrm{H}}$ is a specification of the preferences of the voters in $\underset{\sim}{M}, i . \tilde{e}$. a function $\phi: \underset{\sim}{\mathbb{M}} \rightarrow L(A)$. Let $\Phi$ be the set of all possible profiles for the given $A$. For any $\sigma \varepsilon L(A)$ and $\phi \varepsilon \Phi, n_{\sigma}(\phi)$ will denote the number of voters in $\phi$ having preference order $\sigma$.

A preference function (PF) is a function $f$ that associates with each profile $\phi \varepsilon \Phi$ a nonempty set of preference orders $f(\phi) \subseteq L(A)$ representing the consensus preference orders for the given electorate. (Normally, if there are no ties, $f(\phi)$ will be a unique preference order.) A choice function (CF) is a function $g$ from $\Phi$ to the nonempty subsets of $A ; g(\phi)$ represents the "winning" (i.e. the most preferred) alternative(s) for the given electorate. To each $\mathrm{PF} f$ there corresponds in a natural way a $\mathrm{CF} \hat{\mathrm{f}}$ defined by

$$
\hat{f}(\phi)=\left\{a_{i} \varepsilon A: a_{i} \text { is most preferred for some } \sigma \varepsilon f(\phi)\right\} \text {. }
$$


If $f(\phi)$ depends only on the numbers $n_{\sigma}(\phi)$ for each $\phi$, then $f$ is said to be anonymous. Similarly, if $f$ is symetric in its treatment of alternatives, then $f$ is neutral. Both of these are standard assumptions since Arrow [1], and a PF (or a CF) satisfying both is said to be symmetric [14].

\section{CONSISTENCY AND A GENERALIZATION OF BORDA}

Borda's "order of merit" method may be generalized to the situation where the scores awarded to alternatives are not necessarily evenly spaced. In general, given $\underset{\sim}{s}=\left(s_{1}, s_{2}, \ldots\right.$, $\left.s_{m}\right) \in \mathbb{R}^{m}$ and profile $\phi$ we may assign a score of $s_{m}$ to each voter's least-preferred alternative, a score of $s_{m-1}$ to each next-to-theleast preferred alternative, and so forth. If the total score of alternative $a_{i}$ is denoted by $s_{i}(\phi)$, then we may define a PF $\stackrel{\mathbf{S}}{\sim}$ by

$$
\left(a_{i_{1}}, a_{i_{2}}, \ldots, a_{i_{m}}\right) \varepsilon f^{S}(\phi) \quad \text { iff } \quad s_{i_{1}}(\phi) \geq s_{i_{2}}(\phi) \geqq \cdots \geq s_{i_{m}}(\phi)
$$

Moreover, if ties occur relative to $\underset{\sim}{s}$ (i.e., if several distinct preference orderings result from (2)) then we might use another scoring vector $t \in \mathbb{R}^{\mathrm{m}}$ to resolve these ties; thus we define the composition $\mathrm{f}_{\sim}^{\mathrm{t}}$ of $\mathrm{f}^{\mathrm{S}}(\phi)$ by

$$
\left(a_{i_{1}}, a_{i_{2}}, \ldots, a_{i_{m}}\right) \in f^{t} \circ f^{s}(\phi) \text { iff } s_{i_{1}}(\phi) \geqq s_{i_{2}}(\phi) \geqq \cdots \geqq s_{i_{m}}(\phi)
$$

and for all $\mathrm{k}<\ell, \mathbf{s}_{i_{k}}(\phi)=\mathbf{s}_{i_{\ell}}(\phi)$ implies $t_{i_{k}}(\phi) \geqq t_{i_{\ell}}(\phi)$.

Any PF $f^{\mathbb{S}}$ (or composed series of such functions) and the corresponding $C F$ is called a scoring function $[14,16]$. Borda's method is a particular scoring function in which no provision is made for resolving ties; another example is the method of plurality voting (take $s=(1,0, \ldots, 0))$.

A natural property of voting rules, which in particular is enjoyed by scoring rules, is the following. If two committees 
meeting separately arrive at the same consensus ordering (using some $f)$, then meeting together this should still be their consensus. Hore precisely, we say that a PF (or a CF) $f$ is consistent $[14,15,16]$ if whenever $\phi$ and $\phi^{\prime}$ are profiles on disjoint electorates such that $\mathrm{f}(\phi) \cap \mathrm{f}\left(\phi^{\prime}\right) \neq \phi$; then $\mathrm{f}\left(\phi+\phi^{\prime}\right)=\mathrm{f}(\phi) \cap \mathrm{f}\left(\phi^{\prime}\right)$. (Here $\phi+\phi^{\prime}$ is the profile defined by the union of the two electorates.) Thus, if ties occur in the separate committees, then consensus rankings consist precisely of the ties common to both. It can be shown that any scoring PF $f$ is consistent, as is the corresponding $C F \hat{f}$. In fact, consistency turns out to be the fundamental property characterizing scoring functions when regarded as choice functions.

(4) Theorem 1 [16]. A choice function is a scoring function if and only if it is symmetric and consistent.

Notice, however, that if $f$ is a consistent preference function, then $\hat{f}$ is not necessarily a consistent choice function. For example, with three alternatives $a_{1}, a_{2}, a_{3}$, it is possible that two committees agree on their first choice (say $a_{1}$ ) but differ on their ranking of the remaining two; in this situation consistency for a preference function makes no requirements on the consensus preference order for the two committees together, while a consistent choice function requires that the first choice for the merged committees be $a_{1}$.

Intuitively, consistency for preference functions seems to be a less stringent requirement than consistency for choice functions. However it should also be noticed that this is not, strictly speaking, a weaker concept, since it is possible that $\hat{\mathrm{f}}$ is consistent while $\mathrm{f}$ is not. For example, define the PF $f$ on three alternatives such that $\sigma=\left(a_{i_{1}}, a_{i_{2}}, a_{i_{3}}\right) \varepsilon f(\phi)$ iff $a_{i_{1}}$ has the highest Borda score, and $n_{\sigma}(\phi)^{2}+n_{\tau}(\phi)^{2} \geq n_{\sigma}(\phi)^{2}$ $+n_{\tau},(\phi)^{2}$ where $\sigma^{\prime}=\left(a_{i_{1}}, a_{i_{3}}, a_{i_{2}}\right), \tau=\left(a_{i_{2}}, a_{i_{3}}, a_{i_{1}}\right)$, and $\tau^{\prime}=\left(a_{i_{3}}, a_{i_{2}}, a_{i_{1}}\right)$. 


\section{THE CONDORCET PROPERTY}

Given a profile $\phi$, let $\mathrm{n}_{i j}(\phi)$ be the number of voters preferring $a_{i}$ to $a_{j}$ minus the number preferring $a_{j}$ to $a_{i}$. Condorcet's proposal was that any alternative $a_{i}$ such that $n_{i j}(\phi)>0$ for all $j \neq i$ (i.e., that would defeat any other alternative in pairwise simple majority voting) should be the choice of the group, or, in the case of a preference function, the most-preferred alternative. A parallel concept is that if $a_{i}$ is an alternative such that $n_{i j}(\phi)<0$ for all $j \neq i$ (i.e., it would be defeated by every other alternative), then $a_{i}$ should be the leastpreferred alternative. Suppose now that $a_{i}$ is an alternative such that $n_{i j}(\phi)=0$ for all $j \neq i$. We call $a_{i}$ a quasi-Condorcet alternative for $\phi$. (Fishburn [8] uses this term somewhat differently.) By a natural extension of Condorcet's principle, a quasi-Condorcet alternative is evidently no better than, and also no worse than, any other alternative.

Formally, we say that a PF $f$ is quasi-Condoreet if for any $\phi \varepsilon \Phi$ and any quasi-Condorcet alternative $a_{i}$ for $\phi$

$$
\left(\ldots a_{j}, a_{i} \ldots\right) \in f(\phi) \text { iff }\left(\ldots a_{i}, a_{j}, \ldots\right) \varepsilon f(\phi) \text { for all } j \neq i
$$

In general, a Condoreet alternative is any alternative $a_{i}$ such that $n_{i j}(\phi) \geqq 0$ for all $j \neq i$. We say that a PF $f$ is Condorcet if

$$
\mathrm{n}_{i j}(\phi)>0 \text { implies not }\left(\ldots a_{j}, a_{i} \ldots\right) \in \mathrm{f}(\phi)
$$

and

$$
\begin{aligned}
\mathrm{n}_{i j}(\phi)= & 0 \text { implies }\left(\ldots a_{i}, a_{j} \ldots\right) \in f(\phi) \text { iff }\left(\ldots a_{j}, a_{i} \ldots\right) \\
& \varepsilon f(\phi) .
\end{aligned}
$$

Similarly, we say that a choice function $g$ is Condoreet if $g(\phi)$ is precisely the set of Condorcet alternatives whenever such exist. (This definition corresponds to what Fishburn [7] calls a "strongly Condorcet" CF.) Further, it should be noted that the condorcet property defined above for preference 
functions is somewhat stronger than that used in [12].)

Examp ze 2 .

Consider the following profile on two voters and three alternatives.

$$
\phi: \quad \begin{aligned}
& \left(a_{1}, a_{2}, a_{3}\right) \\
& \left(a_{2}, a_{3}, a_{1}\right)
\end{aligned}
$$

where $a_{1}$ is a quasi-Condorcet alternative. If $f$ is any PF such that $a_{2}$ is always preferred to $a_{3}$ in a collective preference, then for $f$ to be Condorcet we must have

$$
f(\phi)=\left\{\left(a_{1}, a_{2}, a_{3}\right),\left(a_{2}, a_{1}, a_{3}\right),\left(a_{2}, a_{3}, a_{1}\right)\right\}
$$

that is, $a_{1}$ is as good--and as bad--as any other alternative.

A very special case of the Condorcet situation occurs whenever all voters have the same preference order, say $\sigma$. Then $\sigma$ is a Pareto ordering and a PF is said to be pareto if it always chooses the Pareto ordering when one exists. (A still weaker condition, which implies the Pareto property in the presence of consistency, is the following: a PF is faithful if the consensus order is the same as the individual order when society consists of a single individual. This concept was first introduced in [15].)

We say that a PF (or a CF) $f$ is consistent on a subdomain $\Phi^{\prime} \subseteq \Phi$ if whenever $\phi^{\prime}, \phi^{\prime \prime} \varepsilon \phi^{\prime}$, and $\phi^{\prime}+\phi^{\prime \prime}$ is defined and contained in $\Phi^{\prime}$; then

(8) $f\left(\phi^{\prime}\right) \cap f\left(\phi^{\prime \prime}\right) \neq \phi$ implies $f\left(\phi^{\prime}+\phi^{\prime \prime}\right)=f\left(\phi^{\prime}\right) \cap f\left(\phi^{\prime \prime}\right)$. 
Let $\Gamma$ be the subdomain of profiles for which a condorcet alternative exists, that is

(9) $\Gamma=\left\{\phi \varepsilon \Phi: n_{i j}(\phi) \geqq 0\right.$ for some $i$ and all $\left.j \neq i\right\}$.

It is easy to see that any Condorcet choice function is consistent on the domain $\Gamma$. One of the difficulties in trying to find a natural way to extend condorcet's principle to the domain $\Phi-\Gamma$ (which is nonempty if $\mathrm{m} \geq 3$ ) is that it is impossible to find such an extension which is consistent as a choice function.

Theorem 2. There is no condorcet choice function $g$ and domain $\Delta ¥ \Gamma$ such that $g$ is consistent on $\Delta$.

Proof.t Let $\mathrm{m}$ be the number of alternatives. For $\mathrm{m}=1$ or $2, \Gamma=\Phi$ and there is nothing to prove.

Consider then the case $m \geq 3$, and let $\Delta \underset{\ddagger}{\supset}, \phi^{*} \varepsilon \Delta-\Gamma$. Suppose, by way of contradiction, that there exists a condorcet, consistent choice function $g$ defined on $\Delta$. Without loss of generality, let $a_{1} \varepsilon g\left(\phi^{*}\right)$. Since $\phi^{*} \not \Gamma_{,} a_{1}$ is not a condorcet alternative, and there is a $j \neq 1$ such that $n_{j 1}\left(\phi^{*}\right)>0$; say without loss of generality $j=2$. Let $n$ be the number of voters in $\phi^{*}$. Define a new profile $\phi$ on $2 n+n_{21}\left(\phi^{*}\right)$ voters such that $n+n_{21}\left(\phi^{*}\right)$ voters have preference order $\left(a_{1}, a_{2}, a_{3}, \ldots, a_{m}\right)$ and $n$ voters have preference order $\left(a_{2}, a_{1}, a_{3}, \ldots, a_{m}\right)$. Evidently $a_{1}$ is a condorcet alternative for $\phi$, so $\phi \varepsilon \Gamma$ and $y(\phi)=a_{1}$. On the other hand, $\left(\phi^{*}+\phi\right) \in \Gamma$ and $g\left(\phi^{*}+\phi\right)=\left\{a_{1}, a_{2}\right\}$. But consistency implies that $g\left(\phi^{*}+\phi\right)=\left\{a_{1}\right\}$, a contradiction. Notice, moreover, that if $\Delta$ is homogeneous (i.e. $\phi^{*}+\phi^{*}=2 \phi^{*} \varepsilon \Delta$ ), then

$$
g\left(2 \phi^{*}\right)=g\left(\phi^{*}\right)=\left\{a_{1}\right\},
$$

whereas $2 \phi^{*}+\phi \varepsilon \Gamma$ and $g\left(2 \phi^{*}+\phi\right)=\left\{a_{2}\right\}$, contradicting an even weaker version of consistency.

\footnotetext{
TThe authors wish to thank Bengt Hansson for suggesting this
} simple way of proving Theorem 2 . 
(10) Corollary. For $m \geq 3$, no scoring $C F$ is Condorcet.

Theorem 2 does not imply, however, that consistency for preference functions is incompatible with Condorcet's principle. We shall in fact show that there is a preference function with these two properties, and that subject to neutrality, it is unique.

\section{THE KEMENY FUNCTION}

As an example of axiomatic techniques applied to the social sciences kemeny [10] introduced a PF (described in greater detail in Kemeny and Snell [11]) that turns out to play a special role here. The problem defined in [10] is that of finding a consensus preference order for alternatives being considered by a group of "experts". The approach is to reduce the problem "to one which is analogous to those of classical statistics" [11, p. 9] by introducing the idea of a distance measure between any two preference orders. The object then is to axiomatize some measure of distance uniquely. This is achieved by the usual sort of geometric conditions (including the triangle inequality), a certain notion of "betweenness", a kind of independence of irrelevant alternatives condition, and by a normalization assumption. The result of these axioms is the distance measure, which can be described as follows.

With each preference order $\sigma \varepsilon L(A)$ associate an $m \times m$ skew symmetric matrix $x^{\sigma}$, whose rows and columns are labelled by the alternatives, such that the $\left(a_{i}, a_{j}\right)$ entry, $x_{i j}^{\sigma}$, is 1 if $a_{i}$ is preferred to $a_{j}$ in $\sigma,-1$ if $a_{j}$ is preferred to $a_{i}$, and 0 if $i=j \cdot x^{\sigma}$ is called the election matrix for $\sigma$. similarly, if $\phi$ is any non-null profile, the election matrix for $\phi$ is defined to be

$$
\mathrm{x}^{\phi}=\sum_{\sigma} \mathrm{n}_{\sigma}(\phi) \mathrm{x}^{\sigma}
$$

If $\phi$ is null then set $x^{\phi} \equiv 0$. Notice that the $\left(a_{i}, a_{j}\right)$ entry of $x^{\phi}$ is precisely $n_{i j}(\phi)$. 
The Kemeny distance between any two permutations $\sigma$ and $\tau$ is given by

$$
d(\phi, \tau)=\frac{1}{2} \sum_{i, j}\left|x_{i j}^{\sigma}-x_{i j}^{\tau}\right|
$$

The difficulty with this approach is: having achieved the distance measure, it is not clear how to use it to find a consensus. In other words, given a profile $\phi$, what is the ordering that can be said to be "least-distant" from the individuals" orderings in $\phi$ ? Kemeny proposes two alternate solutions from statistics: the median and the mean.

Given $\phi$, a median ranking is defined to be any $\tau$ such that

$$
\tau \operatorname{minimizes} \sum_{\sigma} \mathrm{n}_{\sigma}(\phi) \mathrm{d}(\sigma, \tau) .
$$

A mean ranking is any $\tau$ such that

$$
\tau \text { minimizes } \sum_{\sigma} \mathrm{n}_{\sigma}(\phi) \mathrm{d}^{2}(\sigma, \tau) \text {. }
$$

The reader may verify that in Example 1 the mean is $\left(a_{2}\right.$, $a_{1}, a_{3}$ ) (the same as the Borda result), whereas the median is $\left(a_{1}, a_{2}, a_{3}\right)$.

Kemeny left the problem of which solution to choose unresolved. But from the standpoint of collective decision making there is ample reason to prefer the median, since it turns out that the median consensus leads to a condorcet method, while the mean does not. The latter statement follows from Example 1. To see the former, we re-express the Kemeny function in the following way. For any preference orders $\sigma$ and $\tau$,

$$
d(\sigma, \tau)=\frac{1}{2}\left(x^{\sigma} \cdot x^{\sigma}-x^{\sigma} \cdot x^{\tau}\right)
$$

where, in general, $x \cdot x^{\prime}$ is defined by $x \cdot x^{\prime}=\sum_{i, j} x_{i j} x_{i j}^{\prime} ;$ 
hence, since $x^{\sigma} \cdot x^{\sigma}=m(m-1)$ for any $\sigma$, minimizing $d(\sigma, \tau)$ with respect to $\tau$ is equivalent to maximizing $x^{\sigma} \cdot x^{\tau}$. Thus for any profile $\phi, \tau$ is a median if and only if

$$
\tau \operatorname{maximizes} \sum_{\sigma} n_{\sigma}(\phi) x^{\sigma} \cdot x^{\tau}=x^{\phi} \cdot x^{\tau} .
$$

Kemeny's ruze is the PF defined by

$$
K(\phi)=\left\{\tau: X^{\phi} \cdot X^{\tau}=\max \right\} .
$$

If for some $i$ and $\phi, n_{i j}(\phi)>0$, then we could not have $\tau=\left(\ldots a_{j}, a_{i} \ldots\right) \varepsilon K(\phi)$ because $\tau^{\prime}=\left(\ldots a_{i}, a_{j} \ldots\right)$ satisfies $x^{\phi} \cdot x^{\tau^{\prime}}>x^{\phi} \cdot x^{\tau}$ so $x^{\tau}$ did not maximize $x^{\phi} \cdot x^{\tau}$. On the other hand, if $\mathrm{n}_{i j}(\phi)=0$ and $\tau \varepsilon K(\phi)$ then clearly $\tau^{\prime} \varepsilon K(\phi)$. Hence $\mathrm{K}$ is a Condorcet $\mathrm{PF}$ and, in particular, a quasi-Condorcet $P F$. Notice that $a_{i}$ is a Condorcet alternative for $\phi$ if and only if the $a_{i}$ th row of $x^{\phi}$ is nonnegative. If $a_{i}$ is the unique Condorcet alternative (i.e., the $a_{i}{ }^{\text {th }}$ row of $x^{\phi}$ is positive except for position $\left.\left(a_{i}, a_{i}\right)\right)$ then $a_{i}$ is necessarily most-preferred in any preference order in $K(\phi)$. The fact that Kemeny's median rule is Condorcet was not mentioned by Kemeny, though it provides an excellent reason to prefer the median over the mean. This fact was first pointed out in [12], where a characterization of Kemeny's rule for two and three alternatives was given in terms of six properties. In this paper we shall extend and generalize these results by showing that, for any number of alternatives, the Kemeny rule is the unique preference function that is neutral, consistent and Condorcet, thus reconciling the consistency aspect of Borda's rule with Condorcet's principle.

We have already noted above that $\mathrm{K}$ is Condorcet; it is also immediate that it is symmetric. We claim that $K$ is consistent (as a PF). Indeed, if $\phi^{\prime}+\phi^{\prime \prime}$ is defined and if there is a $\tau$ that maximizes both $x^{\phi^{\prime}} \cdot x^{\tau}$ and $x^{\phi "} \cdot x^{\tau}$, then any such $\tau$ maximizes $x^{\phi^{\prime}+\phi^{\prime \prime}} \cdot x^{\tau}=\left(x^{\phi^{\prime}}+x^{\phi^{\prime \prime}}\right) \cdot x^{\tau}$ as well. Moreover, for any other $\tau^{\prime}$ such that $\left(x^{\phi^{\prime}}+x^{\phi^{\prime}}\right) \cdot x^{\tau}=\left(x^{\phi^{\prime}}+x^{\phi^{\prime}}\right) \cdot x^{\tau}$, ve must have $x^{\phi^{\prime}} \cdot x^{\tau}=x^{\phi^{\prime}} \cdot x^{\tau}$ and $x^{\phi^{\prime \prime}} \cdot x^{\tau^{\prime}}=x^{\phi^{\prime \prime}} \cdot x^{\tau}$, proving that 


$$
\mathrm{K}\left(\phi^{\prime}+\phi^{\prime \prime}\right)=\mathrm{K}\left(\phi^{\prime}\right) \cap \mathrm{K}\left(\phi^{\prime \prime}\right)
$$

whenever the latter is nonempty.

Before proving that these conditions uniquely characterize $K$, we turn to a consideration of the polytope whose extreme points are the election matrices $x^{\sigma}, \sigma \varepsilon L(A)$. This polytope turns out to have important applications to a variety of combinatorial optimization problems [17].

\section{PERMUTATION POLYTOPES}

Let

$$
\stackrel{P}{\sim}=\left\{\lambda_{\sigma} \mathrm{x}^{\sigma}: \sum_{\sigma \in \mathrm{L}(\mathrm{A})} \lambda_{\sigma}=1, \lambda_{\sigma} \geq 0\right\} .
$$

To compute $K(\phi)$ for any $\phi$, it suffices to consider the linear programming problem: maximize $\mathrm{X}^{\phi} \cdot \mathrm{x}$ over all $\mathrm{x} \varepsilon \underset{\sim}{\mathrm{P}}$. The optimum extreme points $x^{\sigma}$ give the consensus ranking(s) $\sigma \in \mathrm{K}(\phi)$. $\underset{\sim}{p}$ is a linear transformation of a so-called permutation polytope $[5,17]$ : if $\mathrm{J}$ is the $\mathrm{m} \times \mathrm{m}$ matrix of all 1 ' $\mathrm{s}$, and $\mathrm{I}$ is the $\mathrm{m} \times \mathrm{m}$ identity matrix, then the transformation

$$
X \rightarrow \frac{1}{2}(X+J-I)
$$

defines the permutation polytope $\underset{\sim}{\mathrm{P}}$ ' associated with $\underset{\sim}{\mathrm{P}}$. The set of inequalities defining $\underset{\sim}{P}$ (and hence $\underset{\sim}{\text { ) }}$ ) are not explicitly known (Bowman having proposed an insufficient set in [5]; a counterexample is due to A.J. Hoffman). However, various characterizations of neighbors on these polytopes are known that give some computational assistance in finding optimal extreme points [17].

For any permutation $\sigma$ let $\mathrm{S}(\sigma)$ be the set of all unordered pairs of symbols $\left\{a_{i}, a_{j}\right\}$ such that $\sigma$ reverses the order of $a_{i}$ and $a_{j}$ (relative to the natural order): 


$$
S(\sigma)=\left\{\left\{a_{i}, a_{j}\right\}: i<j \text { and } a_{j} \text { precedes } a_{i} \text { in } \sigma\right\} .
$$

(21) Let $\Gamma_{\sigma}$ be the graph whose vertex set is $S(\sigma)$ such that $\left\{a_{i}, a_{j}\right\} \varepsilon S(\sigma)$ is adjacent to $\left\{a_{i}, a_{k}\right\} \varepsilon S(\sigma), k \neq j$, if and only if $\left\{a_{j}, a_{k}\right\} \not s(\sigma)$.

The following characterization of neighbors is due to Hoffman. (For a proof of this and other facts about permutation polytopes, see $[17]$.

$$
\begin{aligned}
& \mathrm{x}^{\mathrm{e}} \text { and } \mathrm{x}^{\sigma} \text { are neighbors on } \underset{\sim}{\mathrm{P}} \text { if and only if } \Gamma_{\sigma} \text { is } \\
& \text { connected. }
\end{aligned}
$$

\section{THE PRINCIPAL THEOREM}

(23) Theorem 3. Kemeny's rule is the unique preference function that is neutral, consistent, and condorcet.

We shall in fact prove a stronger result. Define, for any $P F F$, the dual of $F,-F$, to be the PF that inverts the orders of $f$ :

$$
\left(a_{i_{1}}, a_{i_{2}}, \ldots, a_{i_{m}}\right) \varepsilon-f(\phi) \Leftrightarrow\left(a_{i_{m}}, a_{i_{m-1}}, \ldots, a_{i_{1}}\right) \in f(\phi) .
$$

The trivial PF, $T(\phi)$, is the PF defined by

$$
T(\phi)=L(A), \text { for all } \phi \in \Phi \text {. }
$$

We shall show that

$$
\text { the only PF's that are neutral, consistent, }
$$$$
\text { and quasi-Condorcet are } \mathrm{T}, \mathrm{K} \text {, and }-\mathrm{K} \text {. }
$$

Theorem 3 follows from this since of the three only $\mathrm{K}$ is Condorcet--in fact, only $K$ is faithful--when $m \geq 2$ (they are all equal when $m=1$ ). 
To prove (26), we begin by establishing two lemmas. Let $\underset{\sim}{x}$ denote the vector space of all $\mathrm{m} \times \mathrm{m}$, real skew-symmetric matrices.

Lemma 1. Let $f$ be a neutral, consistent, quasi-Condorcet $P F$. Then for every $\phi \varepsilon \Phi, f(\phi)$ depends only on the election matrix $\mathrm{x}^{\phi}$. Moreover, where $x_{\sim}^{Q} \subseteq \underset{\sim}{\mathrm{x}}$ is the set of all rational $\mathrm{m} \times \mathrm{m}$ skew-symmetric matrices, there exists a unique extension of $f$ to $x^{Q}$ that is neutral, consistent, and quasi-condorcet.

Proof. The proof of Lemma 1 is similar to results in [15] and [16]. With $f$ as given, suppose $\phi, \phi^{\prime}$ are two profiles on disjoint voter sets such that $\mathrm{x}^{\phi}=\mathrm{x}^{\phi^{\prime}}$. Choose a profile $\psi$ on a voter set disjoint from those of $\phi$ and $\phi^{\prime}$ such that $x^{\phi+\psi}=$ $x^{\phi^{\prime}+\psi}=0$. $f$ quasi-Condorcet implies $f(\phi+\psi)=f\left(\phi^{\prime}+\psi\right)=L(A)$; hence by consistency

$$
f(\phi)=f(\phi) \cap L(A)=f\left(\phi+\left(\psi+\phi^{\prime}\right)\right)=f\left((\phi+\psi)+\phi^{\prime}\right)=L(A) \cap f\left(\phi^{\prime}\right)=f\left(\phi^{\prime}\right) .
$$

From this it follows that whenever $x^{\phi}=x^{\phi^{\prime}}$ then $f(\phi)=f\left(\phi^{\prime}\right)$ (since we can always find $\phi^{\prime \prime}$ disjoint from $\phi$ and $\phi^{\prime}$ such that $\left.x^{\phi}=x^{\phi "}=x^{\phi}\right)$. We may therefore view $f$ as defined on the domain of election matrices. Notice that any matrix in $x^{\mathbb{Q}}$ can be expressed as a rational linear combination of election matrices. Indeed, for any $i \neq j$ the profile with two voters having preference orders $\left(a_{i}, a_{j}, a_{1}, a_{2}, \ldots a_{m}\right)$ and $\left(a_{m}, a_{m-1}, \ldots\right.$, $\left.a_{1}, a_{i}, a_{j}\right)$ has $n_{i j}=-n_{j i}=2$, and $n_{h k}=0$ for all other $h, k$, and any matrix in $x^{Q}$ can be expressed as a rational linear combination of these. For any positive integer $n>0$ and election matrix $x$ define $f((1 / n) x)=f(x)$. This is well-defined, because if $(1 / n) X=\left(1 / n^{\prime}\right) X^{\prime}$ then by consistency $f\left(n X^{\prime}\right)=f\left(X^{\prime}\right)$ and $f\left(n^{\prime} X\right)=$ $f(x)$; so $f((1 / n)) X)=f\left(\left(1 / n^{\prime}\right) X^{\prime}\right)$. It is easy to verify that this extended $f$ is neutral, consistent, and quasi-condorcet, and this is clearly the only extension of $f$ to $x^{Q}$ that has these properties.o 
A particular consequence of the hypotheses of Lemma 1 is that $f$ is anonymous, hence symmetric. In the sequel, any $f$ satisfying the three hypotheses (in particular, K) will be considered as acting in the domain $\mathrm{x}^{\mathrm{Q}}$.

For the next lemma we need to introduce the notion of derived PF's. For any subset $B \subseteq A,|B|=k>0$, and $\sigma \in L(B)$, let $\sigma\langle\mathrm{A}-\mathrm{B}\rangle$ denote the set of all $\mathrm{m} ! / \mathrm{k}$ ! linear orderings of $\mathrm{A}$ that agree with $\sigma$ when restricted to $B$. Further, let $x_{B}^{Q}$ denote the set of all rational $k \times k$ skew-symetric matrices whose rows and columns are indexed by the elements of $B$, and for any $\mathrm{Y} \varepsilon \underset{\sim \mathrm{B}}{\mathrm{X}}$ let $\mathrm{X}=\mathrm{Y}\langle\mathrm{A}-\mathrm{B}>$ denote the matrix in $\underset{\sim}{\mathrm{X}}$ obtained from $\mathrm{Y}$ by adjoining a zero row and a zero column for every a $\varepsilon A-B$. For any $P F$ defined on $x_{\sim}^{Q}$, let $f^{B}$, the derived $P F$ on alternative set $B$, be defined as follows: For every $Y \in X_{\sim}^{Q}, \quad \sigma \in \mathrm{f}^{\mathrm{B}}(\mathrm{Y})$ iff $\sigma<\mathrm{A}-\mathrm{B}>\subseteq \subseteq \mathrm{f}(\mathrm{Y}<\mathrm{A}-\mathrm{B}>)$.

Lemma 2. If $f$ is a neutral, consistent, quasi-condorcet $\mathrm{PF}$ for alternative set $A$, then

(i) for any $B \subseteq A,|B|>0, f^{B}$ is neutral, consistent, and quasi-Condorcet;

$$
\begin{array}{ccc}
\text { either } & \mathrm{f}^{\mathrm{B}} \text { is Pareto for } a l l|\mathrm{~B}| \geqq 2, \\
\text { or } & -\mathrm{f}^{\mathrm{B}} \text { is Pareto for } a l l|\mathrm{~B}| \geqq 2, \\
\text { or } & \mathrm{f}^{\mathrm{B}} \text { is trivial for } a l l|\mathrm{~B}| \geqq 2 .
\end{array}
$$

Proof. The verification of (i) is left to the reader; it may also be found in [13]. (ii) proceeds by showing that it holds for all $B, 2 \leqq|B| \leqq k$, inducting $k$.

Let $B=\left\{a_{1}, a_{2}\right\}$, and let $x$ be the $2 \times 2$ election matrix corresponding to the preference order $\left(a_{1}, a_{2}\right)$. Then there are three possibilities: 


$$
\begin{aligned}
& f^{\left\{a_{1}, a_{2}\right\}}(x)=\left\{\left(a_{1}, a_{2}\right)\right\}, \\
& f^{\left\{a_{1}, a_{2}\right\}}(x)=\left\{\left(a_{2}, a_{1}\right)\right\}, \\
& f^{\left\{a_{1}, a_{2}\right\}}(x)=\left\{\left(a_{1}, a_{2}\right),\left(a_{2}, a_{1}\right)\right\} .
\end{aligned}
$$

If (28) holds then by neutrality $\mathrm{f}^{\left\{\mathrm{a}_{1}, \mathrm{a}_{2}\right\}}$ is Pareto; if (29) holds, then by neutrality $-f^{\left\{a_{1}, a_{2}\right\}}$ is Pareto; if (30) holds, then by neutrality $\mathrm{f}^{\left\{\mathrm{a}_{1}, \mathrm{a}_{2}\right\}}(-\mathrm{X})=\mathrm{f}^{\left\{\mathrm{a}_{1}, \mathrm{a}_{2}\right\}}(\mathrm{X})$ and since all elements in $\underset{\sim B}{\mathrm{X}}$ are expressible as nonnegative linear combinations of $\mathrm{X}$ and $-X$ it follows by consistency that in this case $f^{\left\{a_{1}, a_{2}\right\}}$ is trivial. Thus (ii) holds for the particular $B=\left\{a_{1}, a_{2}\right\}$; hence by neutrality it holds whenever $|\mathrm{B}|=2$.

Suppose now that (ii) has been shown to hold whenever $2 \leqq$ $|\mathrm{B}| \leqq \mathrm{k}$ for some $\mathrm{k}<\mathrm{m}$; we will show it holds whenever $2 \leqq|\mathrm{~B}| \leqq$ $\mathrm{k}+1$.

Let $B=\left\{a_{1}, a_{2}, \ldots, a_{k+1}\right\}$, and for each $h, 1 \leqq h \leqq k+1$ let $\mathrm{Y}^{\mathrm{h}}=\left(\mathrm{y}_{i j}^{\mathrm{h}}\right)$ be the $(\mathrm{k}+1) \times(\mathrm{k}+1)$ skew-symmetric matrix such that the $h^{\text {th }}$ row and column are zero and

$$
\begin{aligned}
y_{i j}^{h} & =1 \text { if } i<j \quad i, j \neq h \\
& =-1 \text { if } i>j \quad i, j \neq h .
\end{aligned}
$$

(Here the $i^{\text {th }}$ row and column are associated with alternative $a_{i}$, etc.)

$\mathrm{Y}^{\mathrm{h}}$, with the $\mathrm{h}^{\text {th }}$ row and column deleted, is the election matrix $z^{h} \in \underset{\sim}{X_{B}-\left\{a_{h}\right\}}$ corresponding to the preference order $\left(a_{1}\right.$ ' $\left.a_{2}, \ldots, \hat{a}_{h}, \ldots, a_{k+1}\right)=\sigma_{h}$. In the above notation

$$
\mathrm{Y}^{\mathrm{h}}=\mathrm{z}^{\mathrm{h}}<\mathrm{a}_{\mathrm{h}}>\text {. }
$$


Case 1. Suppose (28) holds.

Then by induction $\mathrm{f}^{\mathrm{B}-\left\{\mathrm{a}_{\mathrm{h}}\right\}}$ is pareto, so

$$
f^{B-\left\{a_{h}\right\}}\left(z^{h}\right)=\left\{\sigma_{h}\right\} \quad \text { for all } h, \quad 1 \leqq h \leqq k+1 .
$$

and

$$
f^{B}\left(Y^{h}\right)=\sigma_{h}<a_{h}>
$$

By consistency of $\mathrm{f}^{\mathrm{B}}$,

$$
f^{B}\left(\sum_{h=1}^{k+1} Y^{h}\right)=\bigcap_{1 \leqq h \leqq k+1} \sigma_{h}<a_{h}>=\{\sigma\} .
$$

But

$$
\sum_{h=1}^{k+1} \mathrm{Y}^{\mathrm{h}}=\mathrm{kx} \mathrm{x}^{\sigma}
$$

where $x^{\sigma}$ is the $(k+1) \times(k+1)$ election matrix corresponding to $\sigma$; hence by consistency

$$
f^{B}\left(x^{\sigma}\right)=\{\sigma\} .
$$

Thus by neutrality $f^{B}$ is Pareto for $B=\left\{a_{1}, a_{2}, \ldots, a_{k+1}\right\}$; hence $\mathrm{f}^{\mathrm{B}}$ is Pareto whenever $|\mathrm{B}|=\mathrm{k}+1$. Therefore (28) implies that $\mathrm{f}^{\mathrm{B}}$ is Pareto whenever $2 \leqq|\mathrm{~B}| \leqq \mathrm{k}+1$.

Case 2. If (29) holds, we can apply the same argument as above to show that $-f^{B}$ is pareto for $2 \leqq|B| \leqq k+1$.

Case 3. If (30) holds, applying a similar argument as Case 1 shows that $f^{B}\left(x^{\sigma}\right)=L(B)$ for all permutations of $B=\left\{a_{1}, a_{2}, \ldots, a_{k+1}\right\}$; hence by consistency $f^{B}$ is trivial. Therefore, in fact, $f^{B}$ is trivial whenever $2 \leqq|B| \leqq k+1.0$

A subset $C \subseteq Q^{n}$ ( $Q$ is the set of rationals) is said to be Q-convex if for all $\underset{\sim}{\mathrm{x}}, \underset{\sim}{\mathrm{Y}} \in \mathrm{C}$ and rational $\lambda, 0 \leqq \lambda \leqq 1, \lambda \underset{\sim}{\mathbf{x}}+$ $(1-\lambda) y \in C$. 
One of the peculiar difficulties in proving results about consistent preference functions (and $\mathrm{CF}^{\prime} \mathrm{s}$ ) is that, while under various natural conditions, it is easy to extend their domain uniquely to the rationals (i.e. to include "fractional" voters) in such a way that consistency and the other conditions are still satisfied. (There is in general no immediate way to obtain such an extension to the reals, even though in certain cases a unique extension may exist.) (See for example $[15,16]$.) As a practical matter errors can develop if, during the proofs, one is not careful to restrict the analysis to $Q$-convex sets and rationalvalued vectors. Once the desired characterization is obtained then the extension to the reals is usually obvious. (On this point a difficulty is encountered in certain results in [12], where for example Theorem 2 asserts incorrectly that a certain class of functions may be uniquely extended to the reals.)

To be able to work with Q-convex sets conveniently in the present context, we need the following results. (Proofs may be found in [16]).

Lemma 3 [16]. $C \subseteq Q^{n}$ is Q-convex if and only if

$\underline{C}=Q^{n} \cap$ cvx $C$, where cvx $C$ is the convex hull of $C$.

Lemma 4 [16]. If $C \subseteq Q^{n}$ is $Q$-convex then $c l(C)$ is convex.

Lemma 5 [16]. If $\mathrm{C}=\bigcup_{i=1}^{k} S_{i}$, where $C \subseteq \mathbb{R}^{n}$ is convex and $k$ is finite, then for some $i, \operatorname{dim} C=\operatorname{dim} S_{i}$.

A particular consequence of Lemma 4 is that $C \subseteq c v x C \subseteq c l C$, hence $c \ell C \subseteq c \ell(c v x C) \subseteq c \ell(c \ell C)$ so

$$
c \ell(\mathrm{cvx} C)=\mathrm{c} \ell \mathrm{C} \text {. }
$$

From this we deduce $r i(c \ell c)=r i(\operatorname{cvx} C)$, so by Lemma 3, $Q^{n} \cap r i(c \ell C) \subseteq C$, and $Q^{n} \cap r i(c \ell(C))$ is dense in $r i(c \ell(C))$.

Proof of (26). Let $\mathrm{f}$ be a neutral, consistent, quasiCondorcet SPF. To show that $f$ is $K,-K$, or $T$, it suffices, by Lemma 2, to assume that $f$ is Pareto and to show that $f=k$. (If $f$ is not Pareto then either $-f$ is Pareto or else $f=T$.) 
The proof that $\mathrm{f}=\mathrm{K}$ is by induction on the number of alternatives, $\mathrm{m}$.

If $\mathrm{m}=1$ there is nothing to prove.

Suppose that $m=2$. Then, by Lemma $1, \hat{f}$ is symmetric and consistent; hence (for two alternatives) the associated choice function $\hat{f}$ is also symmetric and consistent, so Theorem 1 implies that $\hat{\mathrm{f}}$ is a scoring function. But clearly the only distinct scoring functions on two alternatives are $f^{(1,0), f(0,1)}$, and $f^{(0,0)}$, which are the same as $K,-K$, and $T$ respectively for two alternatives.

Suppose that $m=3$. For any $x \in x^{Q}, x=\left(x_{i j}\right)$, such that $e=\left(a_{1}, a_{2}, a_{3}\right) \varepsilon K(x)$. It is easy to see that the following inequalities must hold:

$$
x_{12}, x_{23} \geqq 0
$$

$$
\begin{aligned}
& x_{13}+x_{23} \geqq 0 \\
& x_{12}+x_{13} \geqq 0 .
\end{aligned}
$$

Now any $3 \times 3$ rational skew-symmetric $x$ satisfying (31) must be a positive, rational, linear combination of the five skewsymmetric $3 \times 3$ matrices $x^{1}, \ldots, x^{5}$ determined by

$$
\begin{aligned}
& (0,0,0): x^{1} \\
& (1,0,0): x^{2} \\
& \left(x_{12}, x_{13}, x_{23}\right)=(0,1,0): x^{3} \\
& (0,0,1): x^{4} \\
& (1,-1,1): x^{5}
\end{aligned}
$$

$$
\text { We claim that } f\left(x^{i}\right)=K\left(x^{i}\right) \text { for } 1 \leqq i \leqq 5 \text {. }
$$

Indeed $f\left(X^{1}\right)=K\left(x^{1}\right)=L\left(a_{1}, a_{2}, a_{3}\right)$ by neutrality; also, $\left.f\left(x^{2}\right)=\left(a_{1}, a_{2}\right)<a_{3}\right\rangle=\left\{\left(a_{1}, a_{2}, a_{3}\right),\left(a_{1}, a_{3}, a_{2}\right),\left(a_{3}, a_{1}, a_{2}\right)\right\}=K\left(x^{2}\right)$. 
by the fact that $f^{B}$ is pareto for all $|B| \geq 2$ and $f$ is quasicondorcet. Similarly $f\left(x^{3}\right)=k\left(x^{3}\right), f\left(x^{4}\right)=k\left(x^{4}\right)$. Finally, note that $x^{5}$ is symmetric under any 3 -cycle permutation of $a_{1}$, $a_{2}$, and $a_{3}$; hence (since $f\left(x^{5}\right) \neq \phi$ and if is neutral) either

$$
f\left(x^{5}\right)=\left\{\left(a_{1}, a_{2}, a_{3}\right),\left(a_{2}, a_{3}, a_{1}\right),\left(a_{3}, a_{1}, a_{2}\right)\right\}
$$

or

$$
f\left(x^{5}\right)=\left\{\left(a_{3}, a_{2}, a_{1}\right),\left(a_{2}, a_{1}, a_{3}\right),\left(a_{1}, a_{3}, a_{2}\right)\right\}
$$

or

$$
f\left(x^{5}\right)=L\left(\left\{a_{1}, a_{2}, a_{3}\right\}\right)
$$

Now

$$
Y=X^{\left(a_{2}, a_{1}, a_{3}\right)}=\left[\begin{array}{rrr}
0 & -1 & 1 \\
1 & 0 & 1 \\
-1 & -1 & 0
\end{array}\right]
$$

and because $f$ is Pareto, $f(Y)=\left\{\left(a_{2}, a_{1}, a_{3}\right)\right\}$. But $Y+X^{5}=2 x^{4}$, so if either (34) or (35) were true consistency would imply $f\left(X^{4}\right)=f(Y) \cap f\left(X^{5}\right)=\left\{\left(a_{2}, a_{1}, a_{3}\right)\right\}$, a contradiction. Therefore $f\left(x^{5}\right)$ is as in (33), to $f\left(x^{5}\right)=K\left(x^{5}\right)$ and $(32)$ holds.

Now for any $x$ such that $K(x)=\{e\}$ we have $x=\sum_{i} \lambda_{i} x^{i}$ for some rational $\lambda_{i}>0$; so by consistency and the fact that $\mathrm{e} \varepsilon \mathrm{f}\left(\mathrm{x}^{\mathrm{i}}\right) \stackrel{\mathrm{i}}{=} \mathrm{K}\left(\mathrm{x}^{\mathrm{i}}\right)$ for all $\mathrm{i}$,

$$
f(x)=\bigcap_{i} f\left(x^{i}\right)=\bigcap_{i} k\left(x^{i}\right)=k(x) .
$$

Thus $f(X)=K(X)$ whenever e $\varepsilon K(X)$; hence by symmetry $f(X)=$ $K(X)$ for all $X$, proving the theorem for $m=3$.

Suppose that $m \geqq 4$. The domain of $f$ and of $k$ is $\mathrm{X}_{\sim}^{\mathrm{Q}} \subseteq \underset{\sim}{\mathrm{X}}$, and, by consistency, the sets

$$
\mathrm{f}_{\sigma}^{-1}=\left\{\mathrm{x} \in \underset{\sim}{\mathrm{\sim}} \mathrm{x}^{\mathrm{Q}}: \sigma \varepsilon \mathrm{f}(\mathrm{X})\right\}
$$

are Q-convex for each $\sigma \varepsilon L(A)$. 
Similarly

$$
\mathrm{K}_{\sigma}^{-1}=\{\mathrm{X} \in \underset{\sim}{\mathrm{X}} \underset{\sim}{Q}: \sigma \varepsilon \mathrm{K}(\mathrm{X})\}
$$

is Q-convex for each $\sigma \in L(A)$.

Note that $\mathrm{f}_{\sigma}^{-1} \supseteq \mathrm{f}^{-1}(\sigma)=\left\{\mathrm{X} \in \underset{\sim}{\mathrm{X}^{Q}}: \mathrm{f}(\mathrm{X})=\{\sigma\}\right\}$ and $\mathrm{k}_{\sigma}^{-1} \supseteq \mathrm{K}^{-1}(\sigma)=$ $\left.\mathrm{X} \in \underset{\sim}{\mathrm{X}^{Q}}: \mathrm{K}(\mathrm{X})=\{\sigma\}\right\}$.

By Lemma $4, c \ell\left(f_{\sigma}^{-1}\right)=\overline{f_{\sigma}^{-1}}$ is convex for each $\sigma$. Since

$\bigcup_{\sigma} \overline{f_{\sigma}^{-1}}=\underset{\sim}{x}$, Lemma 5 implies that int $\overline{\mathrm{f}_{\sigma}^{-1}} \neq \phi$ for some $\sigma$. hence

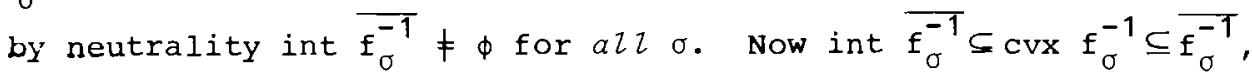
so by Leruna 3 ,

$$
\mathrm{x}_{\sim}^{Q} \cap \text { int } \overline{\mathrm{f}_{\sigma}^{-1}} \subseteq \mathrm{f}_{\sigma}^{-1}
$$

We claim that

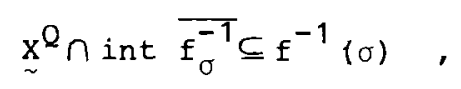

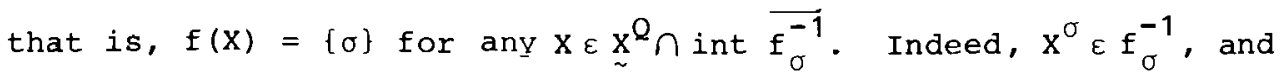

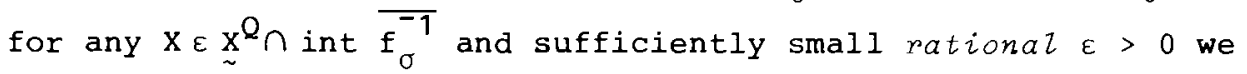
have $\mathrm{Y}=(1+\varepsilon) \mathrm{X}-\varepsilon \mathrm{X}^{\sigma} \varepsilon \mathrm{X}_{\sim}^{Q} \cap$ int $\overline{\mathrm{f}_{\sigma}^{-1}}$ and

$$
X=\left(\frac{\varepsilon}{1+\varepsilon}\right) X^{\sigma}+\left(\frac{1}{1+\varepsilon}\right) Y \quad ;
$$

so by consistency

$$
f(X)=f\left(X^{\sigma}\right) \cap f(Y)=\{\sigma\},
$$

proving (39). (39) that

$$
\text { Since } x_{\sim}^{Q} \text { is dense in int } \overline{f_{\sigma}^{-1}} \text { for all } \sigma \text {, it follows from }
$$

$$
\text { int } \overline{\mathrm{f}_{\sigma}^{-1}} \cap \text { int } \overline{\mathrm{f}_{\tau}^{-1}}=\phi \quad \text { for all } \sigma \neq \tau \text {. }
$$


Therefore by the separation theorem for convex sets there exists, for every $\sigma \neq \tau$, a nonzero matrix $U^{\sigma \tau} \varepsilon \underset{\sim}{X}$ such that

$$
\begin{aligned}
& \mathrm{U}^{\sigma \tau} \cdot \mathrm{x} \geqq 0 \quad \text { for all } \mathrm{x} \in \overline{\mathrm{f}_{\sigma}^{-1}} \\
& \mathrm{U}^{\sigma \tau} \cdot \mathrm{x} \leq 0 \quad \text { for } \mathrm{all} \mathrm{x} \in \overline{\mathrm{f}_{\tau}^{-1}} .
\end{aligned}
$$

Here "•" denotes the inner product of $\mathrm{u}^{\sigma \tau}$ and $\mathrm{x}$, regarded as vectors.

Let $e=\left(a_{1}, a_{2}, \ldots, a_{m}\right) \in L(A)$ denote the identity permutation, and let $\sigma$ be any neighbor of $e$ (i.e., $x^{\sigma}$ is a neighbor of $\mathrm{x}^{\mathrm{e}}$ on the polytope $\left.\mathrm{P}_{\sim}\right)$. Let $\mathrm{U}^{\mathrm{e} \sigma}=\left(u_{i j}\right)$ and recall from section 5 that $S(\sigma)$ is the set of pairs $p=\left\{a_{i}, a_{j}\right\}$ such that $i<j$ and $a_{j}$ precedes $a_{i}$ in $\sigma$. We claim that for some $\lambda>0$ and for all $i<j$,

$$
u_{i j}=-u_{j i}=\lambda \text { whenever }\left\{a_{i}, a_{j}\right\} \in S(\sigma)
$$

$$
u_{i j}=-u_{j i}=0 \text { whenever }\left\{a_{i}, a_{j}\right\} \not L s(\sigma) .
$$

First, given any pair $\left\{a_{i}, a_{j}\right\} \not S(\sigma)$, let $i<j$ and define a matrix $x \in \underset{\sim}{X^{Q}}$ by $x_{i j}=-x_{j i}=1$, and 0 elsewhere. Since $f^{\left\{a_{i}, a_{j}\right\}}$ is Pareto, the quasi-condorcet property of $f$ implies that $\mathrm{e}, \sigma \in \mathrm{E}(\mathrm{X})$; hence $\mathrm{U}^{\mathrm{e}} \cdot \mathrm{X}=0$ and

$$
u_{i j}=0 \text { whenever }\left\{a_{i}, a_{j}\right\} \notin s(\sigma) .
$$

Now let $\mathrm{p}=\left\{\mathrm{a}_{\mathbf{i}}, \mathrm{a}_{j}\right\}, \mathrm{q}=\left\{\mathrm{a}_{\mathbf{i}}, \mathrm{a}_{\mathrm{k}}\right\} \in \mathrm{S}(\sigma)$ be any adjacent pairs in the graph $\Gamma_{\sigma}$, where, without loss of generality, $j<k$. Then $\left\{\mathbf{a}_{j}, \mathbf{a}_{\mathbf{k}}\right\} \notin \mathbf{S}(\sigma)$ and either

or

$$
e=\left(\ldots a_{i} \ldots a_{j} \ldots a_{k} \ldots\right) \text { and } \sigma=\left(\ldots a_{j} \ldots a_{k} \ldots a_{i} \ldots\right) \text {, }
$$$$
e=\left(\ldots a_{j} \ldots a_{k} \ldots a_{i} \ldots\right) \text { and } \sigma=\left(\ldots a_{i} \ldots a_{j} \ldots a_{k} \ldots\right) \text {. }
$$ 
Define a matrix $x=\left(x_{i j}\right) \varepsilon \underset{\sim}{x^{Q}}$ such that

(44)

$$
\begin{aligned}
& x_{i j}=-x_{j i}=1, \\
& x_{j k}=-x_{k j}=1, \\
& x_{i k}=-x_{k i}=-1, \\
& x_{h \ell}=0 \quad \text { for all other } h, l .
\end{aligned}
$$

Letting $B=\left\{a_{i}, a_{j}, a_{k}\right\}$ we know by Lemma 2 that $f^{B}$ satisfies our hypotheses, so if $\hat{x}$ is $x$ restricted to the rows and the columns corresponding to $a_{i}, a_{j}, a_{k}$, then $\left(a_{i}, a_{j}, a_{k}\right) \varepsilon f(\hat{x})$ and $\left(a_{j}, a_{k}, a_{i}\right)$ $\varepsilon f(\hat{X})$, by the preceding analysis for $m=3$ (i.e. (33) above). since $f$ is quasi-Condorcet, we therefore have $e, \sigma \in f(X)$; whence $\mathrm{u}^{\mathrm{e} \sigma} \cdot \mathrm{x}=0$ and

$$
2 u_{i j}+2 u_{j k}-2 u_{i k}=0
$$

By (43), $u_{j k}=0$; whence

$$
\begin{aligned}
& u_{i j}=u_{i k} \text { whenever }\left\{a_{i}, a_{j}\right\} \in S(\sigma) \text { is adjacent } \\
& \text { in } \Gamma_{\sigma} \text { to }\left(a_{i}, a_{k}\right) \in S(\sigma) \text {. }
\end{aligned}
$$

(46) Notice, moreover, that if $\left\{a_{i}, a_{j}\right\} \in S(\sigma)$ is adjacent in $\Gamma_{\sigma}$ to $\left\{a_{i}, a_{k}\right\} \in S(\sigma)$, then $i<j$ implies $i<k$ (because if not then we have $e=\left(\ldots a_{k} \ldots a_{i} \ldots a_{j} \ldots\right)$ so $\sigma=\left(\ldots a_{j} \ldots a_{i} \ldots a_{k} \ldots\right)$ and $\left\{a_{j}, a_{k}\right\} \in S(\sigma), a$ contradiction).

Since $\sigma$ is a neighbor of $e, G_{\sigma}$ is connected (22); hence (45) and (46) imply that for some $\lambda$

$$
u_{i j}=-u_{j i}=\lambda \text { whenever } i<j \text { and }\left\{a_{i}, a_{j}\right\} \varepsilon s(\sigma)
$$

$$
u_{i j}=u_{j i}=0 \text { whenever } i<j \text { and }\left\{a_{i}, a_{j}\right\} \notin s(\sigma) \text {. }
$$

Since $f$ is Pareto, $x^{e} \varepsilon \mathrm{f}_{\mathrm{e}}^{-1}$, and we must have $\mathrm{u}^{\mathrm{e}} \cdot \mathrm{x}^{\mathrm{e}} \geqq 0$, that is, $\lambda \geqq 0$, and in fact $\lambda>0$ because $U^{e \sigma} \neq 0$, proving (42). 
Without loss of generality, let $\lambda=1$; then

$$
U^{e \sigma}=\frac{1}{2}\left(x^{e}-x^{\sigma}\right) \text {. }
$$

For any given $\mathrm{Y} \varepsilon \mathrm{X}^{\mathrm{Q}}$, if $\mathrm{e} \varepsilon \mathrm{f}(\mathrm{Y})$ then

$$
\mathrm{U}^{\text {eo }} \cdot \mathrm{Y} \geq 0 \text {, that is, }
$$

$$
X^{e} \cdot Y \geq X^{\sigma} \cdot Y \text { for all neighbors } \sigma \text { of } e \text {. }
$$

It follows that

$$
\mathrm{X}^{\mathrm{e}} \cdot \mathrm{Y} \geq \mathrm{X}^{\tau} \cdot \mathrm{Y} \quad \text { for all } \tau \in \mathrm{L}(\mathrm{A})
$$

implying that e $\varepsilon K(Y)$. Thus e $\varepsilon f(Y)$ implies e $\varepsilon K(Y)$, so by neutrality $f$ is a refinement of $\mathrm{K}$, that is,

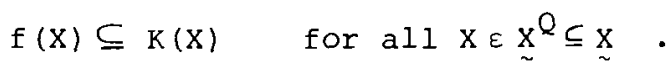

We claim that

(52) whenever $\sigma$ is a neighbor of $\tau, \sigma, \tau \varepsilon K(X)$ and $\tau \varepsilon f(X)$ imply $\sigma \varepsilon f(X)$.

Suppose $(52)$ is false for some neighbors $\sigma$ and $\tau$. Say without loss of generality that $\tau=e$. Then for some $x^{0} \varepsilon{\underset{\sim}{\alpha}}^{Q}$,

$$
\sigma, e \in K\left(x^{\circ}\right), \quad \text { e } \varepsilon f\left(x^{\circ}\right), \quad \sigma \notin f\left(x^{0}\right) .
$$

Since $0 \varepsilon \mathrm{K}_{\mathrm{e} \sigma}^{-1}=\{\mathrm{X} \varepsilon \underset{\sim}{\mathrm{X}}: \mathrm{e}, \sigma \in \mathrm{K}(\mathrm{X})\}, \underset{\sim}{\mathbb{F}}=$ aff $\mathrm{K}_{\mathrm{e}}^{-1}$ is a subspace of $x$. Let

$$
\begin{aligned}
& {\underset{\sim}{\mathrm{e}}}_{\mathrm{e}}=\mathrm{f}_{\mathrm{e}}^{-1} \cap \mathrm{K}_{\mathrm{e} \sigma}^{-1}, \\
& \underset{\sim}{\mathrm{Y}_{\sigma}}=\mathrm{f}_{\sigma}^{-1} \cap \mathrm{K}_{\mathrm{e \sigma}}^{-1} .
\end{aligned}
$$

Further, let $\underset{\sim}{\bar{Y}}=c \ell(\underset{\sim}{\mathrm{Y}}) \subseteq \underset{\sim}{\bar{Y}}, \underset{\sim}{\overline{\mathrm{Y}}}=\mathrm{c} \ell(\underset{\sim}{\mathrm{Y}}) \subseteq \underset{\sim}{\mathrm{Y}}$. Since $\mathrm{e}$ and $\sigma$ are neighbors, there is an $X \varepsilon \underset{\sim}{X}$ such that

$$
x \cdot x^{e}=x \cdot x^{\sigma}>x \cdot x^{\pi} \quad \text { for all } \pi \neq e, \sigma .
$$

Hence there is a rational $x^{*} \varepsilon \underset{\sim}{X^{Q}}$ with this property, and 


$$
K\left(X^{*}\right)=\{e, \sigma\} .
$$

Let $W=r i\left(\operatorname{cvx} \mathrm{K}_{\mathrm{eo}}^{-1}\right)$. For every rational $\mathrm{x} \in \underset{\sim}{W}$ and sufficiently small rational $\lambda>0, \mathrm{X}^{*} \varepsilon \operatorname{cvx} \mathrm{K}_{\mathrm{e} \sigma}^{-1}$ implies

$$
x^{\prime}=(1+\lambda) x-\lambda x^{*} \varepsilon \underset{\sim}{W}
$$

$X$ rational implies $X^{\prime} \varepsilon k_{e \sigma}^{-1}$ (see tile remarks after Lemma 5); hence $\{e, \sigma\} \subseteq K\left(X^{\prime}\right) \cap K\left(X^{*}\right)=K(X)$. But $K\left(X^{*}\right)=\{e, \sigma\}$; so

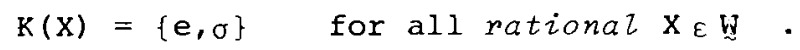

For any rational $X \in \underset{\sim}{W},(51)$ combined with (54) shows that

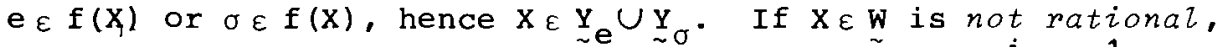
then $x$ is a limit point of a sequence of rational $x^{i} \varepsilon k_{e \sigma}^{-1}$. Hence there is a subsequence in $\underset{\sim}{\mathrm{Y}}$ e converging to $X$, or a subsequence in $\underset{\sim}{Y}$ converging to $X$. In any case

$$
\underset{\sim}{\bar{Y}} \cup \underset{\sim \sigma}{\cup} \underset{\sim}{\underline{W}}
$$

By Lemma 5, at least one of the subspaces aff $\underset{\sim}{\bar{Y}_{e}}$, aff $\underset{\sim}{\bar{Y}} \sigma$ is equal to $\underset{\sim}{\bar{Y}}$; let the other be $\underset{\sim}{\bar{Y}} \cdot \subseteq \underset{\sim}{\bar{Y}}$.

we claim that

$$
\operatorname{ri} \underset{\sim}{\bar{Y}} e \cap r i{\underset{\sim \sigma}{Y}}_{\sigma}=\phi .
$$

Suppose (56) is false. Then $r i \underset{\sim}{\underset{Y}{\mathrm{Y}}} \cap \mathrm{ri} \underset{\sim}{\bar{Y}_{\sigma}}=S \neq \phi$ is open with respect to the subspace $\bar{\sim}^{\prime}$ '. Since every point in $\underset{\sim}{\bar{Y}}$ is a limit point of $\underset{\sim}{Y} \subseteq \underset{\sim}{X^{Q}}$, and similarly for $\underset{\sim}{\bar{Y}}{ }_{\sigma}, S$ contains $a$ rational $X^{\prime} \varepsilon \underset{\sim}{X^{Q}}$, and by the remarks after Lemma 5 ,

$$
X^{\prime} \varepsilon \underset{\sim}{Y} e^{\cap} \underset{\sim}{Y} \sigma
$$

The $x^{0}$ of $(53)$ is in ${\underset{\sim}{Y}}_{e^{\prime}}$ so for sufficiently small rational $\lambda>0$, 


$$
\mathrm{X}^{\prime \prime}=(1+\lambda) \mathrm{X}^{\prime}-\lambda \mathrm{x}^{0} \in \operatorname{ri} \underset{\sim \mathrm{P}}{\overline{\mathrm{Y}}},
$$

whence e $\varepsilon f\left(X^{\prime \prime}\right)$, Since

$$
x^{\prime}=x^{n} /(1+\lambda)+\lambda x^{0} /(1+\lambda),
$$

f consistent implies

$$
\text { e } \varepsilon f\left(x^{\prime}\right)=f\left(x^{\prime \prime}\right) \cap f\left(x^{\circ}\right),
$$

while

$$
\sigma \notin f\left(x^{0}\right) \text { implies } \sigma \notin f\left(X^{\prime}\right)
$$

Thus $X^{\prime} \not \underset{\sim}{Y}{ }_{\sigma^{\prime}}$ contradicting (57). This shows that $(56)$ is true.

The separation theorem for convex sets implies that there is a nonzero skew-symmetric real matrix $U, U \in \underset{\sim}{\mathrm{Y}}$, such that

$$
\begin{array}{ll}
\mathrm{U} \cdot \mathrm{X} \geqq 0 & \text { for all } \mathrm{X} \in \underset{\sim \mathrm{e}}{\overline{\mathrm{Y}}_{\mathrm{e}}}, \\
\mathrm{U} \cdot \mathrm{X} \leqq 0 & \text { for all } \mathrm{X} \in \underset{\sim \sigma}{\overline{\mathrm{Y}}_{\sigma}} .
\end{array}
$$

Then, just as in the derivation of (47), we conclude that for some real number $\lambda$,

$$
\begin{aligned}
& u_{i j}=-u_{j i}=\lambda \text { whenever } i<j \text { and }\left\{a_{i}, a_{j}\right\} \in s(\sigma), \\
& u_{i j}=-u_{j i}=0 \text { whenever } i<j \text { and }\left\{a_{i}, a_{j}\right\} \not s(\sigma) .
\end{aligned}
$$

But $\quad U \in \underset{\sim}{\bar{Y}}=\operatorname{aff} K_{e \sigma}^{-1}$ implies $U \cdot\left(x^{e}-x^{\sigma}\right)=4 \sum_{i<j} u_{i j}=0$.

$$
\left\{a_{i}, a_{j}\right\} \in S(\sigma)
$$

Thus $\lambda=0$, so $U=0$, contradicting the choice of $U$. This proves (52).

Now suppose $f \neq K$, that is, by (51) there is an $X^{*}$ such that $\mathrm{f}\left(\mathrm{X}^{*}\right) \underset{\neq}{\subsetneq} \mathrm{K}\left(\mathrm{X}^{*}\right)$. Say

$$
\sigma, \tau \in \mathrm{K}\left(\mathrm{X}^{*}\right), \sigma \in \mathrm{f}\left(\mathrm{X}^{*}\right), \tau \notin \mathrm{f}\left(\mathrm{X}^{*}\right)
$$


Let ${\underset{\sim}{\mathrm{P}}}^{*}$ be the polytope $\left\{\mathrm{X} \varepsilon \underset{\sim}{\mathrm{P}}: \mathrm{X}^{*} \cdot \mathrm{X}=\max \right\}$.

Since $\left|K\left(X^{*}\right)\right| \geq 2$, dim $P^{*} \geq 1$, so by a theorem of Balinski [2] there is a path $\sigma=\pi^{1}, \pi^{2}, \ldots, \pi^{k}=\tau$ such that for $1 \leq i<k$, $\mathrm{x}^{\pi^{i}}$ is a neighbor of $\mathrm{X}^{\pi^{i+1}}$ on $\underset{\sim}{\mathrm{P}}$ and $\pi^{i} \varepsilon \mathrm{K}^{*}$ ) for $1 \leq i \leq k$. By (52), $\sigma=\pi^{1}, \pi^{2} \varepsilon \mathrm{K}\left(\mathrm{X}^{*}\right)$ and $\tilde{\sigma}=\pi^{1} \varepsilon \mathrm{f}\left(\mathrm{X}^{*}\right)$ implies $\pi^{2} \varepsilon \mathrm{f}\left(\mathrm{X}^{*}\right)$ and so forth; we conclude that $\pi^{k}=\tau \varepsilon f\left(X^{*}\right)$, a contradiction. Thus $f$ is identical to K. $\mathrm{K}$

\section{CONCLUSION}

In the social choice literature two divergent streams of thought are apparent. One, which stems from Borda's work, emphasizes scoring methods; the fundamental property enjoyed by these methods is that they are consistent, which amounts to saying that they satisfy a kind of Pareto principle for subgroups. This condition seems very natural and desirable in the context of collective decision making, and it is difficult to see how a preference function not satisfying this property would be able to find general acceptance. The other, which grows out of Condorcet's proposal that an alternative able to get a majority over any other should be the most-preferred alternative, is the problem of extending this idea in a natural way to cases when a Condorcet alternative does not exist.

As we have shown, these two ideas, suitably interpreted for preference functions, are resolved in the method known as kemeny's rule; moreover subject to the basic requirement that all alternatives be treated in an unbiased manner, Kemeny's rule is the only preference function satisfying the three properties.

The present development, compared to Kemeny's, also provides an interesting example of the use of axioms in the social sciences. Kemeny axiomatized a distance measure by invoking certain mathematically appealing properties. But by concentrating on the distance minimizing aspect of the problem, the point was missed that it is the decision function itself, rather than the distance, that possesses the important properties. 


\section{REFERENCES}

[1] Arrow, K.J., Social Choice and Individual Values, John Wiley, New York, 1963.

[2] Balinski, M.L., On the Graph Structure of Convex Polyhedra in n-space, Pacific Journal of Mathematics, 11 (1961), $431-434$.

[3] Black, D., The Theory of Committees and Elections, Cambridge University Press, Cambridge, England, 1958.

[4] de Borda, J.C., Mémoire sur les Élections au Scrutin, Histoire de l'Académie Royale des Sciences, Paris, 1781.

[5] Bowman, V.J., Permutation Polyhedra, SIAM Jour. Appl. Math., $(1972), 580-589$.

[6] Condorcet, M.J.A.N.C., Marquis de, Essai sur Z'Application de l'Analyse à la Probabilité des Décisions Rendues à la Pluralité des Voix, Paris, 1785.

[7] Fishburn, P.C., The Theory of Social Choice, Princeton University Press, Princeton, N.J., 1973. - Condorcet Social Choice Functions, memeographed, Pennsylvania State University, 1976.

[9] Gilmore, P.C. and A.J. Hoffman, A Characterization of Comparability Graphs and of Interval Graphs, Can. Jour. Math. , $16(1964), 539-548$.

[10] Kemeny, J., Mathematics Without Numbers, Daedalus, 88 (1959), 577-591.

[11] and L. Snell, Mathematical Models in the Social Sciences, Ginn and Co., Boston, 1960.

[12] Levenglick, A., Fair and Reasonable Election Systems, Behavioral Science, $\underline{20}$ (1975), 34-46. - Characterizations of Social Decision Functions, $\mathrm{Ph}$.D. Thesis, Graduate School of the City University of New York, New York, 1977.

[14] Young, H.P., A Note on Preference Aggregation, Econometrica, $\underline{42}(1974), 1129-1131$.

, An Axiomatization of Borda's Rule, Jour. Ec. Theory, $\underline{9}(1974), 43-52$. 
[16] Young, H.P., Social Choice Scoring Functions, SIAM Jour. Appl. Math., 28 (1975), 824-838.

[17] On Permutations and Permutation Polytopes, to appear in Mathematical Programming. 